\title{
Genuine Modal Realism, the Humean thesis and advanced modalizing
}

\author{
Sonia Roca-Royes ${ }^{1}$ (D)
}

Received: 23 August 2014 / Accepted: 8 February 2018 / Published online: 19 March 2018

(C) The Author(s) 2018

\begin{abstract}
The paper argues that Lewis' Genuine Modal Realism, in taking the plurality of worlds to be necessarily the way it is, implies the existence of necessary connections of the sort that contradicts the Humean thesis that Lewis endorses. By endorsing, pace Divers, a non-redundancy interpretation of advanced modalizing, we gain the means to exactly state what these connections amount to.
\end{abstract}

Keywords Advanced modalizing · Genuine Modal Realism · Humean thesis · Lewis · Possible worlds

We can always ask the modal question "Is it possible that reality is different from that postulated by the realist". [...] the reasonable answer is 'yes'.

(Skyrms 1976, p. 332)

\section{Introduction}

Modalizing can be, in Divers's terms, basic or advanced. Basic modalizing is modalizing about ordinary individuals; where, in the context of (Divers 1999), the notion of an ordinary individual is characterized as that of individuals that not only are spatiotemporally located but also have all their (spatiotemporal) parts spatiotemporally related to one another. By contrast, advanced modal claims

are modal claims about entities other than spatiotemporally unified individuals (perhaps, then, spatiotemporally disunified individuals, sets, numbers, properties, propositions and events). (Divers 1999, p. 217)

Sonia Roca-Royes

sonia.rocaroyes@stir.ac.uk

1 University of Stirling, Room A74, Pathfoot, Stirling FK9 4LA, Scotland, UK 
Skyrms's question above-like his answer-is an advanced modal one. The use of advanced modalizing is very common too when raising objections of moral indifference against Lewis's Genuine Modal Realism; 'GMR' henceforth. These are objections unified in the first instance by the fact that they explore the consequences of Lewis's claim that, contrary to Skyrms's reasonable expected answer, "the character of the totality of worlds is not a contingent matter" (Lewis 1986, p. 126); a claim which is itself an instance of advanced modalizing. ${ }^{1}$ They are then unified also by the fact that they find the consequences of this Lewisian claim untenable, leading to moral indifference.

Thus, Adams asks, on the basis of the necessary character of the totality of worlds, "What is wrong with actualizing evils, since they will occur in some other possible world anyway if they don't occur in this one?" (Adams 1974, p. 216) and complains that GMR cannot provide an ethically satisfying answer. Reviving the spirit of Adams' indifference objection, Heller argues that there's no reason to save a drowning child when the saving of her is inevitably linked—due again to the necessary character of the totality of worlds - to the dying of another drowning child: "What is morally relevant to the indifference is the known inevitability of one survival and one death no matter which choice is made" (Heller 2003, p. 9). In a similar vein, Beedle's indifference concerns stem also from the fact that the totality of worlds could not be different. In his case, however, somewhat more contentiously, the concerns depend on assuming that that necessity implies that the character of each world is necessary too: "If the character of all the worlds is given, then the character of each world is given" (Beedle 1996, p. 492).

Regardless of whether we are persuaded by these objections, neither Adams nor Heller nor Beedle can be accused of making an illegitimate use of advanced modalizing. Rather, they are exploring the consequences of one of several of Lewis' own instances of advanced modalizing. It is not trivial, however, what interpretation is to be given, in GMR, to advanced modal claims. As Heller notes, commenting on his claim that the saving of a drowning child (in one world) is inevitably linked to the dying of another drowning child (in another world), "it is not easy to cash out the sense of inevitability involved" (Heller 2003, p. 15). This is an obstacle to the clear statement of any objection to GMR that rests on the use of advanced modal claims. The literature has gone in two directions. Hudson (1997, 1999), wondering about the modal status of GMR itself, opts for interpreting advanced modal claims in the same way as basic modal claims (thus not making any explicit distinction between the two types of modalizing): "possibly $\mathrm{P}$ if and only if at some world $\mathrm{W}, \mathrm{P}$ is true at $\mathrm{W}$ - where the phrase 'at $\mathrm{W}$ ' serves to restrict the domain of quantification of quantifiers in $\mathrm{P}$ to the parts of W" (Hudson 1997, p. 80). Since GMR expresses a transworld state of affairs, is it true at no word (given the enforced restricted interpretation of the quantifiers occurring in GMR) and, therefore, as Hudson notes, GMR is, on such interpretation, if true, necessarily false; a result that, he claims, should not bother Lewis. Unlike Hud-

\footnotetext{
1 Equivalent advanced modal claims by Lewis are: "There is but one totality of worlds; it is not a world, it could not have been different" (Lewis 1986, p. 80; my emphasis); "It is futile to want the entire system of worlds to satisfy a condition, it is not contingent what conditions the entire system of worlds does or does not satisfy." (Lewis 1986, p. 125).
} 
son, Divers proposes, "on behalf of the genuine modal realist" (Divers 1999, p. 217), a redundancy interpretation of advanced modalizing: whenever $p$ expresses a transworld state of affairs, both 'it is possible that $p$ ' and 'it is necessary that $p$ ' are semantically equivalent to ' $p$ ', making thus the modal expressions, only in the context of advanced modalizing, redundant. On this interpretation, GMR is, if true, necessarily true.

Against this dialectical background, the contribution of the present paper is twofold. First, I, too, will be exploring (Sects. 3, 4) the consequences of Lewis's claim that the character of the totality is not a contingent matter. This exploration will bring me to the claim that, in GMR's ontology, there are necessary connections among different existents of the sort Lewis thinks there should be none. These are the same necessary connections (or inevitable links) that Heller identifies and on the basis of which he mounts his moral indifference objection. Compatibly with Heller's, however, my objection will rather be that GMR violates the same Humean thesis it endorses.

Sensitive (though not explicitly) to the fact that the statement of these necessary connections involves advanced modalizing, Heller writes (assuming what seems to be an interpretation of advanced modalizing closer to Hudson's) that "Lewis gives us no good way to describe the necessary connection[s] ... because necessity is truth within all worlds, and the connection in question is inter-world" (Heller 2003, p. 16). The second fold of this paper's contribution is to find the means of describing these necessary connections in a way such that: (i) it is dialectically appropriate, thus not being unfair to Lewis; (ii) the intended claims stating necessary connections come out true, departing from Hudson; (iii) the force the objection that can be mounted on their bases is not pre-empted; departing, as we shall see, from Divers. This will be done (Sect. 5) by suggesting an interpretation of advanced modalizing that is neither Hudson's nor Divers's and that will enable a neat restatement of the tension identified in the first fold. As we shall see, the interpretation will be closer to Divers's yet it will allow us to do proper justice to Hudson's.

\section{Background and terminology}

\subsection{Worlds versus possibilities}

Lewis says-for good reasons - that one should not count possibilities by counting possible worlds. For, as he notes:

A single world may provide many possibilities, since many possible individuals inhabit it. To illustrate, consider these two possibilities for me. I might have been one of a pair of twins. I might have been the first-born one, or the secondborn one. These two possibilities involve no qualitative difference in the way the world is. [...] The haecceitist says: two possibilities, two worlds. They seem just alike, but they must differ somehow. [...] they differ in what they represent, $d e$ $r e$, concerning someone. [...] I say: two possibilities, sure enough. And they do indeed differ in representation de re: according to one I am the first-born twin, 
Table 1 Representation by worlds

\begin{tabular}{lrcc}
\hline Concrete, representing level & $\begin{array}{c}\text { Actual world (@) } \\
\text {..Humphrey loses... }\end{array}$ & $\begin{array}{c}\text { World 1 (w1) } \\
\text {...Humphrey* wins... }\end{array}$ \\
\hline Representation relation & & $\Downarrow$ & $\Downarrow$ \\
\hline Represented possibilities & $@$ & ...Humphrey loses... & ...Humphrey wins... \\
& $\mathrm{w} 1$ & ...Humphrey* loses... & ...Humphrey* wins... \\
\hline
\end{tabular}

according to the other I am the second-born. But they are not two worlds. They are two possibilities within a single world. $(230-231)^{2}$

Unlike what Lewis' illustration might suggest, however, the need for cutting (de re) possibilities finer than worlds does not arise only after admitting the possibility of multiple counterparts within a single world. It arises already once we admit that counterparts of actual things in other worlds are of analogous modal nature as the actual things they are counterparts of-by which we might simply mean: they are equally being represented in virtue of having, too, counterparts. That is, it arises already when we admit that: "The same goes in reverse. Our Humphrey is a counterpart of many Humphreys of many other worlds". (195) A world where a winning counterpart of Humphrey_let's say, Humphrey*-exists represents de re, concerning Humphrey, that he wins the election. But it also represents de re, concerning Humphrey* (and indeed concerning any other individual Humphrey* is a counterpart of) that he wins. Similarly, our world represents that Humphrey loses and also that the winning Humphrey* loses $^{3}$ :

Regardless of whether there are multiple counterparts within a single world, therefore, the existence of other worldly counterparts plus their analogous modal nature already implies that the actual world - as a whole-represents many distinct de re possibilities: it represents at least one for each concrete possible world. ${ }^{4}$ The same goes for any other world in the plurality (Table 1).

What the (theoretical) possibility of there being multiple counterparts within a single world has an impact on is how many de re possibilities a world represents concerning individuals of one same world. Let w2 be a world where there is a pair of individuals, $a$ and $b$, that are counterparts of one another. Let us assume that $a$ is a businessman and that $b$ is a film director. By $a$ and $b$ being counterparts of one another, the representational facts include those pictured in Table 2.

Like Lewis's twins-world example, w2 represents (at least) two distinct de re possibilities concerning individuals of w2. Generalising, any world, wi, that contains individuals $x$ and $y$ such that $a$ and $b$ are each counterparts of both would appear (at least) twice in an appropriate extension of Table 2.

\footnotetext{
2 Unless otherwise stated, bracketed page or section numbers are from Lewis (1986).

3 This example assumes that Humphrey and Humphrey* are counterparts of one another. The example does not commit me-nor is Lewis committed - to the counterpart relation being symmetrical or reflexive; although it surely often is, as Lewis admits (see Lewis 1986, p. 195).

4 This is so because the actual world is a counterpart of each world. In general, each world is a counterpart of each world. (In other words, the counterpart relation restricted to the set (or class) of worlds results in a total, therefore symmetrical, relation.)
} 
Table 2 Worlds vs. possibilities

\begin{tabular}{lll}
\hline Concrete, representing level & \multicolumn{1}{c}{$\begin{array}{c}\text { World } \mathbf{2}(\mathbf{w 2}) \\
\text {...a is a businessman and } \mathbf{b} \text { is a film director... }\end{array}$} \\
\hline Representation relation & & \multicolumn{1}{|c}{} \\
\hline Represented possibilities & $\mathrm{w} 2$ & ... a is a businessman and $\mathrm{b}$ is a film director... \\
& $\mathrm{w} 2$ & ... a is a businessman and $\mathrm{b}$ is a film director... \\
\hline
\end{tabular}

Terminology I will call 'world-possibilities' what worlds, as a whole, represent de re. Whenever I need to be explicit about which world a world-possibility is a possibility for, I will replace 'world' by the name of that world. For instance, that world-possibility represented by w that talks about actual individuals will be called '@-possibility'. Whenever I need to be explicit also that the representing world is w, I willwrite '@-possibility/w'. This should be read as meaning the @-possibility according towhich@is qualitatively isomorphic to the way wis.

\subsection{Qualitative versus de re ways (possibilities)}

The preceding remarks prove it convenient to distinguish the qualitative ways worlds might be from the de re ways. There are correspondingly different kinds of representation:

We can distinguish representation of the way things are qualitatively (in a broad sense of that word) from representation $d e$ re. It may be the case, according to a world, that there are things of certain kinds, arranged in certain ways, and with certain causal relationships; these are matters of qualitative character. But also, it may be the case, according to a world, concerning the individual Humphrey, that he exists and waves and wins. (198)

De re ways-but not (purely) qualitative ways - involve particular individuals. As a result, a de re way a world could be is a way only one world could be. ${ }^{5}$ By contrast, a qualitative way is a way many worlds could be (indeed instantiate). For instance, with respect to the 1968 US presidential election, the de re ways only our world could be are: (Nixon wins \& Humphrey loses) and (Nixon loses \& Humphrey wins); the de re ways only w1 could be are: (Nixon* wins \& Humphrey* loses) and (Nixon*loses \& Humphrey*wins); and the qualitative ways both@ and w1 could be are: ( $x$ wins \& $y$ loses). Given that representation works by qualitative isomorphism, any two de re ways represented by the same world will be the same qualitative way.

\footnotetext{
5 This follows from Lewis' thesis of non-overlap (Sect. 4.2). It is for the same reason that, when commenting on the principle of recombination (on which I turn soon in the main text), Lewis says:

I cannot altogether accept the formulation: anything can coexist with anything. For I think the worlds do not overlap, hence each thing is part of only one of them. A dragon from one world and a unicorn from a second world do not themselves coexist either in the dragon's world, or in the unicorn's world, or in a third world. (88)
} 
Notation As I have just done, I will notationally distinguish a de re possibility from a (purely) qualitative possibility by using (names for) the relevant individuals in the first case, and variables in the second.

\subsection{The principle of recombination and the Humean thesis}

In On the Plurality of Worlds, Lewis struggles, for real difficulties, to offer a precise formulation of the principle of recombination. He offers in Sect. 1.8 some initial remarks but then, as he goes along, he also tells us (especially on page 181) that those initial remarks ought to be generalized. He never, however, offers a formulation of the resulting generalized principle. I'm afraid I cannot do better than him. Yet, since a lot of what follows turns on it, it is important to have enough of a grip on what the principle amounts to. ${ }^{6}$

In general, a principle of recombination tells us what possibilities there are. Lewis's is a Humean principle of recombination: it encodes the Humean "denial of necessary connections between distinct existences" (87). Accordingly, a very rough formulation of the principle is that "anything can coexist with anything else, at least provided they occupy distinct spatiotemporal positions. Likewise, anything can fail to coexist with anything else". (88) A first qualification to be made leads to the introduction of the famous 'size and shape permitting' proviso. The unqualified principle tells us that any number of copies of a thing can coexist with any number of copies of another. But, as Lewis notes, "[O]nly a limited number of distinct things can coexist in a spacetime continuum". (89) This puts an upper bound to the number of copies of a given thing that can coexist within a spacetime unit; hence, the proviso.

For reasons I cannot expand on here, ${ }^{7}$ a second qualification is to the effect that the rough statement above should be read in terms of duplicates: a duplicate of anything can coexist with a duplicate of anything else. With this first approximation to the principle, of the Humean thesis, we are exploiting its denial of "a necessary connection between the intrinsic character of a thing and the intrinsic character of distinct things with which it coexists (181)". The Humean thesis, however, also prohibits

a necessary connection between the intrinsic character of a thing and its external relations to other things. It cannot be, for instance, that there is an absolutely necessary connection (as opposed to a contingent law of nature) whereby every charged particle must be exactly a certain distance from another particle. (181)

And this, as Lewis says, gives us a "companion principle" (181) that, exegetically, we should understand as being subsumed under an accordingly generalized principle of recombination. The upshot of recombination thus generalized is that "spatiotemporal arrangement may vary independently of the intrinsic nature of the things arranged" (181).

There is at least one more level of generalization required; for not all external relations are spatio-temporal ones. For the sake of not anticipating content unneces-

\footnotetext{
6 Thanks to two anonymous referees for this journal for pressing me on this.

7 See (Lewis 1986, p. 88), and also footnote 5 above.
} 
sarily — which would also betray the dialectics of the paper-I shall return to this in due course (in Sect. 4). I shall finish these introductory remarks on recombination by noting that the principle, in GMR, is not a world-making principle. It is not, for instance-and cannot be-a principle that will generate all worlds that there are out of parts of this world. ${ }^{8}$ Rather, we ought to think of it as a principle that is satisfied by the plurality and its represented possibilities; whatever there is in it.

\subsection{The principle of plenitude and its necessity}

The principle of recombination gives Lewis the resources of expressing, nonvacuously, his tenet that there is a plenitude of worlds. The statement of plenitude will rely on a correspondence between concrete worlds and Quinean ersatz worlds. Quinean ersatz worlds are mathematical representations: namely, "set[s] of quadruples of real numbers, regarded as giving the coordinates of the spacetime points that are occupied by matter" (90). The Quinean method, Lewis says,

could be extended to allow for various sizes and shapes of spacetime, for occupancy by different kinds of matter and by point-sized bits of fields, and perhaps even for occupancy of times by non-spatial things. (90)

Once extended to all possible sizes and shapes-something for mathematicians to determine_plenitude gets stated thus: "for every Quinean ersatz world, there is a genuine world with the represented pattern of occupancy and vacancy. This is just an appeal to recombination". (90)

The principle of plenitude plays in GMR the role of ensuring sufficient descriptive power to Lewis' account. It ensures that for any (de re) way our world could be, there is a world that represents our world as being that (de re) way. More generally (and given the analogous modal nature of everything that there is), it ensures that the entire system of worlds is such that for any qualitative way a world could be, there is a world that is that qualitative way.

With a view to having enough descriptive power, the truth of the principle of plenitude suffices. Yet, in GMR, the principle is also said to be necessary. As anticipated in Sect. 1, in one of several places where Lewis engages with advanced modalizing, Lewis attributes the following modal property to the entire system of worlds: "There is but one totality of worlds; it is not a world, it could not have been different" (80, my emphasis). The entire system of worlds, therefore, is necessarily the way it is.

It is well known that this necessary character of the plurality serves several purposes and it helps Lewis strengthen his abductive argument for GMR. It helps Lewis respond to the epistemological objection: "Our contingent knowledge that there are donkeys at our world requires causal acquaintance with the donkeys... Our necessary knowledge that there are donkeys at some worlds... does not". (112). It helps him also respond to some versions of the moral indifference objection: "It is futile to want the entire system of worlds to satisfy a condition, because it is not contingent what conditions the entire

\footnotetext{
8 As Lewis notes (92), alien worlds won't be generated that way.
} 
system of worlds does or doesn't satisfy". (125). Lewis' abduction to GMR would be accordingly weakened if he were to renounce the necessary status of plenitude.

Additionally, the contingency of the principle of plenitude would place Lewis on a par with an Ersatzer who declared that the actual world - the only world that there is according to her-is such that all possible properties are instantiated (or that all possible properties actually exists, whether with instances or not), thereby denying the possibility of alien properties. In both cases, the descriptive power of the respective theories would be maximal thanks to modal luck: of all the ways the world/the totality could be, it would contingently happen to be in a way that allows us to have sufficient descriptive resources. If the Lewisian were to take the plenitude of worlds to be contingent, the ersatzer could — without (straightforward) comparative loss-declare that there could not be swappable alien properties although, relative to some (non-actual) possible world, there could. ${ }^{9}$

Despite the apparent (abductive) benefits of holding that the plenitude of worlds is necessary, it can be shown that such necessity generates the following tension in GMR: It makes the account violate the Humean thesis it endorses. This will be unfolded in Sect. 4, after first spending Sect. 3 clarifying how to understand the Lewisian claim of necessity.

\section{The necessary character of the plurality}

As anticipated in the introduction, Beedle (1996) takes it that the necessary character of the totality implies that the character of each world is necessary too: "If the character of all the worlds is given, then the character of each world is given" (Beedle 1996, p. 492). I believe that GMR, as it's been developed in Lewis (1986), already contains everything needed to resist this conditional claim and this section will unfold the way to do so. To anticipate: compatibly with keeping the qualitative character of the totality constant, there is variability as to which world instantiates which qualitative way a world could be.

That Beedle's reasoning is a non-sequitur is assumed in passages such as the following by Heller, where the last sentence stresses the qualitative constancy of the totality despite the de re variability alluded to in the preceding one:

If Roz saves Righty, Lisa will let Lefty drown, and if Roz lets Righty drown, Lisa will save Lefty. Just as in the other two cases, one child will be saved and one will be drowned. (Heller 2003, p. 11)

The faulty step has also been explicitly noted by Ridenour:

The [antecedent in Beedle's conditional] states only that the totality of possible worlds encompasses all logical possibilities. This does not in itself determine which logical possibility must be enacted by this world. Can I choose to forego another cup of coffee this evening? Ignoring issues surrounding the debate about

\footnotetext{
9 Also, an important methodological question would arise here as to the extent to which theoretical serviceability should allow us to postulate a theory that not only cannot be tested, but also such that its serviceability depends on its contingency.
} 
free will and determinism, I will assume that I can. If I choose to forego another cup of coffee, the [antecedent] only entails that I have a counterpart in some world that will not choose to do so. (Ridenour 2000, p. 112)

We saw in Sect. 2.2 that 'way' can be understood as meaning qualitative way or de $r e$ way; a distinction Lewis introduces also by means of distinguishing the qualitative character (Lewis 1986, p. 198) from what we can call the 'de re character' of a world. So when he says that the character of the totality is not a contingent matter, disambiguation is in order. I shall argue in Sect. 3.1 that, as GMR has been developed in 1986, Lewis has everything he needs to disambiguate this claim by saying that it is only in the qualitative sense that the plurality is necessarily the way it is, having therefore the resources to respond to Beedle's in precisely the way that Ridenour envisions.

\subsection{Pluralities versus possibilities}

Lewis's reasons for cutting (world-)possibilities finer than worlds were seen in Sect. 2.1. We shall see here that, by the same token, one should cut totality-possibilities finer than totalities. I shall motivate this by using a very simple example that will serve as a case-study. Suppose that subject $a$, in the actual world, has a glass, $b$, in her hand which she throws against the floor $(T a b)$. There are two possibilities: that the glass breaks $(B b)$, and that the glass does not break $(\neg B b)$. By the principle of plenitude, there are two concrete worlds each representing one of these possibilities. ${ }^{10}$ Suppose that the glass actually breaks. There is then a concrete world where other concrete entities-say, $c$ and $d$-are such that $d$ is a counterpart of $b$ and $c$ is a counterpart of $a$ and such that $d$ does not break after $c$ throws it against the floor.

Once distinct concrete entities of analogous modal nature ${ }^{11}$ as $a$ and $b$ enter the story, so do distinct de re possibilities, not only concerning them but also their counterparts. As far as representing de re possibilities is concerned, therefore, our concrete entities-which, apart from being represented are also representatives-are polysemic. ${ }^{12}$ Table 3 (top of next page) shows the scenario just described. ${ }^{13}$

In general, any concrete entity $x$ represents de re any other concrete entity $y$, such that $<x, y>$ belongs to the counterpart relation. Thus, $B b$ in our world represents as many de re possibilities as there are individuals $y$ such that $\langle b, y\rangle$ belongs to counterpart relation (Table 3).

\footnotetext{
10 I am assuming, for simplicity, that $b$ has no counterpart in the actual world other than itself.

11 Recall (from Sect. 2): by 'of analogous modal nature' we only mean having counterparts too.

12 We can speak of things as being polysemic since Lewis' language is a particularly rich variant of a Lagadonian language; one where not only (by the reflexivity of the counterpart relation) concrete entities are names of themselves, but also where they are names of other entities: those they are counterparts of.

13 I shall assume in this paper, for simplicity, that the counterpart relation is symmetric.
} 
Table 3 Case-study representation

\begin{tabular}{lrcc}
\hline Concrete, representing level & $\begin{array}{c}\text { Actual world (@) } \\
\text {...Tab \& Bb... }\end{array}$ & $\begin{array}{c}\text { World 3 (w3) } \\
\text {...Tcd \& } \neg \text { Bd... }\end{array}$ \\
\hline Representation relation & & $\Downarrow$ & $\Downarrow$ \\
\hline Represented possibilities & $@$ & $\ldots$ Tab \& Bb... & $\ldots$ Tab \& $\neg$ Bb... \\
& w3 & $\ldots$ Tcd \& Bd... & $\ldots$ Tcd \& $\neg$ Bd... \\
\hline
\end{tabular}

As seen in Sect. 2.1, Lewis had strong motivations to cut possibilities finer than worlds. Furthermore, in the case of multiple counterparts within a world, one world can represent more than one de re possibility concerning individuals of the same world. It was so, for instance, in the case of w2 and also in the case of Lewis's world with a pair of identical twins which are counterparts of one another. Table 2 above illustrated how w2 represents more than one (de re) w2-possibility; which are nonetheless qualitatively the same way.

So it is, I submit, in the case of the totality of worlds. One should not count de $r e$ totality-possibilities by counting totalities. The totality of worlds is an entity-a trans-world one - that contains maximal spatiotemporal units-the worlds - that are counterparts of one another. It is a trans-world entity with multiple counterparts within it. In the language of representation de re, this has, as a consequence, that it represents more than one de re possibility concerning the individuals of one (and the only!) totality of worlds. This is strictly the same type of phenomenon that we saw with world w2, its businessman and its film director. The only difference is one of scale.

There's nothing in GMR that impedes the occurring of this phenomenon at the level of totalities. It does involve us thinking advancedly modally about that large, trans-world individual that the totality is. In particular, it involves us thinking that $i t$ can be de re different even if not qualitatively different. But advanced modal thinking is legitimate from the standpoint of GMR, even in those cases where the plurality itself is the object of our advanced modalizing; indeed, Lewis has been the one to introduce us to such practice.

Apart from there being no impediment to the larger-scale phenomenon, passages like the following, where Lewis addresses indifference objections, explain how natural it is to receive Lewis in exactly the way Heller and Ridenour, and myself, have received him:

The book I want to have written will be written in any case-of necessity, it will be written countless times over, word for word the same. [...] Among my counterparts and myself are many who succeed in writing it, and many who fail. What does it matter where I myself fall? [...] It will not matter to reality as a whole how [I] decide - there will in any case be many just like [me] who decide one way and many who decide the other-but it still matters to [me]. [...] I do not just idly want someone in some world to have written this book I have in mind; I want to have written it myself, and that is what motivates me to keep going. (124-126; my emphasis) 
Table 4 Representation by worlds; generalization

\begin{tabular}{lrccccc}
\hline Concrete, representing level & $@$ & w1 & w2 & w3 & $\ldots$ \\
\hline Representation relation & & $\Downarrow$ & $\Downarrow$ & $\Downarrow$ & $\Downarrow$ & $\ldots$ \\
\hline Represented possibilities & $@$ & @/@ & @/w1 & $@ / w 2$ & $@ / w 3$ & $\ldots$ \\
& w1 & w1/@ & w1/w1 & w1/w2 & w1/w3 & $\ldots$ \\
& w2 & w2/@ & w2/w1 & w2/w2 & w2/w3 & $\ldots$ \\
& w3 & w3/@ & w3/w1 & w3/w2 & w3/w3 & $\ldots$ \\
& $\ldots$ & $\ldots$ & $\ldots$ & $\ldots$ & $\ldots$ & $\ldots$ \\
\hline
\end{tabular}

\subsection{What (de re) possibilities does the totality represent?}

To say that, compatibly with being necessarily the qualitative way it is, the totality can be de re different still leaves open the question what are the de re possibilities for the totality. Consider Table 4, which pictures the plurality and all world-possibilities represented individually by each of its worlds. ${ }^{14}$

Let us focus on the @-column. It goes against GMR to say that the conjunction of all the world-possibilities represented by the actual world (@/@,w1/@,w2/@, w3/@ ,...) could obtain in (is a possibility for) the totality of worlds. Saying that would amount to denying Lewis's claim that the totality of worlds "could not have been different" (80) in both the qualitative and the de re senses. The totality satisfies plenitude, and it satisfies it as a matter of necessity; so it is not a possibility for it that all its worlds are the same qualitative way. But plenitude does not require that the entire system of worlds be such that $w 1$ is the qualitative way it is, and that $w 2$ is the qualitative way it is, and that $\mathrm{w} 3$ is the qualitative way it is, and so on. In other words, the requirements of plenitude are merely existential. As a result, there is more than one combination (without repetition) of world-possibilities that satisfy plenitude; and more than one among those that should be taken as de re possibilities for the totality.

To unfold this by means of our case study from Sect. 3.1, I shall make some further assumptions for expository reasons and generalize afterwards. The first further assumption is that the only contingent fact of the actual world is $B b$ and that its only alternative possibility is $\neg B b$. This assumption implies that there are only two ways the actual world could be. Given these two possibilities, plenitude ensures the existence of two concrete worlds: ${ }^{15}$ the actual world (where $(T a b \& B b)$ ), and w3 (where (Tcd \& $\neg B d$ )). I assume also that also w 3 could only have been in those two qualitative ways. The two assumptions together imply (under the non-essential further assumption that there are no indiscernible worlds) that @ and w3 are the only two worlds in the entire system of worlds. I am then assuming — until I generalize - that the entire system of worlds contains just two worlds.

\footnotetext{
14 In this table and at other places I conveniently abbreviate ${ }^{\ulcorner}$wi-possibility/wj ${ }^{\circ}$ with $\ulcorner\mathrm{wi} / \mathrm{wj}\urcorner$.

$15 \mathrm{I}$ am assuming also, therefore, and also for simplicity reasons, that glass $b$ has no counterpart in the actual world other than itself.
} 
Consider now the two de re possibilities for @—-(Tab\&Bb) and $(T a b \& \neg B b)$-plus the two de re possibilities for w3- $(T c d \& B d)$ and $(T c d \& \neg B d)$. Of the four combinations below, only the first two satisfy plenitude: ${ }^{16,17}$

(i) $\{(T a b \& B b)$ and $(T c d \& \neg B d)\}$

(ii) $\{(T a b \& \neg B b)$ and $(T c d \& B d)\}$

(iii) $\{(T a b \& B b)$ and $(T c d \& B d)\}$

(iv) $\{(T a b \& \neg B b)$ and $(T c d \& \neg B d)\}$

Combination (iii) violates plenitude because the @-possibility that (Tab\& $\neg B b$ ), and the w3-possibility that ( $T c d \& \neg B d$ ) would be represented by no world in the entire system of worlds; and similarly for combination (iv). The claim above that the requirements of plenitude are merely existential amounts to saying, with Lewis, that, so long as plenitude is satisfied, "It will not matter to reality as a whole" whether it is @ or w3 that instantiates (Txy \& By) or (Txy \& $\neg$ By). In general terms, so long as plenitude is satisfied, is does not matter to the account which world instantiates which of the qualitative ways for a world to be.

Given that neither (i) nor (ii) violates (under all the assumptions in place) any of Lewis' principles, they should—taking Sect. 3.1 into account-be considered as distinct de re ways the totality of worlds could be. Notice especially that, while being de re different, these combinations are qualitatively the same:

$$
\{(\text { Txy \& By) and (Txy \& } \neg \text { By) }\}
$$

Indeed, both (i) and (ii) are qualitatively isomorphic to the way the totality is. As a result, representation of totality-possibilities works by qualitative isomorphism plus the identity facts as supplied by the counterpart-relational facts. That is, it works exactly like representation of world-possibilities does, according to what Lewis has taught us. The one totality, $T$, manages therefore to represent them both, as pictured in Table 5 (next page).

Consequently, given the necessity of plenitude, such de re contingency of the totality does not call for further totalities. ${ }^{18}$ As noted in Sect. 3.1, this is, at a larger scale, strictly the same phenomenon that we saw with world w2, its businessman and its film director.

So when Lewis reasons that "There is but one totality of worlds; it is not a world, it could not have been different" (80) we can — and should — understand him as having in mind the qualitative way of difference. (We know, from Sect. 2.1, that he is vigilant not to make the mistake of counting de re (or individual) world-possibilities by counting worlds. We can expect him to be equally vigilant at the level of the totality).

\footnotetext{
16 I am writing '( $T a b \& B b)$ ' to refer to the whole @-possibility/@ including that fact; and likewise for the other de re possibilities. This way it is easier to see why only some of them satisfy plenitude. One should keep in mind, however, that I am referring to whole world-possibilities, and not just to the part explicitly mentioned in each case.

17 I am ignoring other combinations that also satisfy plenitude-like $\{($ Tab \& Bb) and (Tab \& $\neg B b)\}$ - because, for obvious reasons, we are interested not only in combinations without repetition but also in combinations that contain exactly one world-possibility for each world.

18 This should alleviate some (not all) of the worries in Skyrms (1976) and Yagisawa (1988).
} 
Table 5 Totality-possibilities

\begin{tabular}{lcc}
\hline Concrete, representing level & Totality \\
& & $\{\ldots$ Tab \& Bb... \& ...Tcd \& $\neg$ Bd ... $\}$ \\
\hline Representation relation & & $\Downarrow$ \\
\hline Represented totality-possibilities & $\mathrm{T}$ & $\{\ldots$ Tab \& Bb.. \& ..Tcd \& $\neg \mathrm{Bd} \ldots\}$ \\
& $\mathrm{T}$ & $\{\ldots$ Tab \& Bb... \& ..Tcd \& $\neg \mathrm{Bd} \ldots\}$ \\
\hline
\end{tabular}

Terminology As already done in Table 5, and by analogy to the term 'worldpossibility', I shall use 'totality-possibility' to refer to the maximal de re possibilities for the totality.

We can then - and should - conceive of the totality of worlds as possibly being in different de re ways. For those with a modal-deflationary appetite, this can simply be taken to mean that, given counterpart-relational facts among worlds, the totality's descriptive power exceeds that of a strictly Lagadonian language. ${ }^{19}$ These (de re) totality-possibilities can be taken as just being admissible conjunctions of worldpossibilities. 'Admissible' as a restriction because, as we have seen, not everything goes. There is a principle that governs which conjunctions of world-possibilities are totality-possibilities and which are not. According to the Lewisian tenets, only the combinations (without repetition) that satisfy (A) are admissible, where (A.1) assumes plenitude and guarantees its necessity:

(A) (A.1) Any qualitative way some world is is instantiated at least once by some world, ${ }^{20}$ and

(A.2) For any world, w, there is exactly one w-possibility in each of them. ${ }^{21}$

\section{Necessary connections among different existences: a problem}

With the preceding stock, I shall here, and by means of modalizing advancedly, state the necessary connections in which, in GMR, different existents stand. I will do this, in the first instance, by focusing on our two-world example from Sect. 3.2. I will then generalize and qualify accordingly. As I will subsequently show, the troublesome nature (for GMR) of these necessary connections is that they are of a sort Lewis thinks there should be none.

Prior to generalizing, I am using our case study with the further assumptions introduced in Sect. 3.2; to the effect that the entire system of worlds consists of only @ and w3. Because the principle of plenitude is not only true but necessarily so, the de re possibilities $B b$ and $B d$ cannot both obtain in the totality of worlds, despite

\footnotetext{
19 See footnote 12 for the precise way in which it exceeds a Lagadonian language.

20 They could appear more than once only if there were indiscernible worlds; something Lewis leaves open.

21 That there should be constraints on which conjunctions are totality-possibilities and which aren't should not be surprising or deemed per se problematic. There are corresponding constraints at the level of worlds. Mirroring (A.2), a world with a pair of twins, $a$ and $b$, that are counterparts of one another does not represent that $a$ is both the first and the second born. What will prove problematic, as per the next section, are the specific constraints Lewis endorses; in particular, (A.1).
} 
involving different existences (even spatio-temporally isolated ones). These two possibilities - in our two-world example - stand in the relation of mutual metaphysical exclusivity; and the same holds for $\neg B b$ and $\neg B d$. Similarly, $B b$ and $\neg B d$ necessitate each other; and so do $B d$ and $\neg B b$. We can put this in terms of world-possibilities. The necessity of plenitude implies that there are pairs of world-possibilities-in our two-world example - that stand in the relation of mutual metaphysical exclusivity. Corresponding to the impossibilities that the totality of worlds be in any of the (iii) or the (iv) de re ways (from Sect. 3.2 above), we have, respectively, that:

$$
\begin{aligned}
& \text { Necessarily } \neg(@ / @ \text { and w3/@) } \\
& \text { Necessarily } \neg(@ / w 3 \text { and w3/w3) }
\end{aligned}
$$

And corresponding to (i) and (ii) we have that:

@/@ andw3/w3 necessitate one another; and

@/w3 andw3/@ necessitate one another

which is to say:

\section{Necessarily [(@/@ andw3/w3) or (@/w3 andw3/@)]}

These (advanced) necessities, when conjoined with the de re (advanced) contingency of the totality, serve some purposes in GMR. First, they make GMR have the (abductive) benefits mentioned in Sect. 2.4. And, contra Beedle, they would do so without grounding modal fatalism-more strongly: being incompatible with it. For, despite the fact that the totality cannot but satisfy plenitude, the character of our world is not fixed: according to some totality-possibilities, Lewis "falls" on the book-finisher side and, according to some others, he doesn't. Second (and consequently), they can also help us make sense of Lewis' response to objections of moral indifference. It might be "futile to want the entire system of worlds to satisfy a [qualitative] condition" (125; '[qualitative]' my addition on the basis of Sect. 3). If I decide to save a drowning child, another one would drown somewhere else. Yet, the totality can be such that $I$ satisfy the de se desire of saving the child. Against what Beedle suggests (1996), our options are not merely to wait to simply discover whether I save the child or not (that is, to discover where we "fall", to use Lewis's term); I can decide to save her and, according to some totality-possibility, I save her.

Despite their theoretical purposes, these necessities are striking. In our two-world example, Lewis is committed to the existence of pairs of world-possibilities concerning different concrete worlds (@ and w3) such that: each of them, taken individually, is (non-advancedly) possible; they involve no common individual; the individuals they involve are said to be spatiotemporally and causally isolated; and still, they unintelligibly stand in the relation of mutual metaphysical exclusivity.

These necessities are striking because they place constraints (as captured by (A.1) above) upon "co-realization of possibilities" 22 which do not match the pre-theoretical constraints. Pre-theoretically, and according to the widely-endorsed compossibility thesis, two processes that do not overlap are not constrained in the way Lewis' theory implies. The realization of the possibility of making a table, $a$, from a given hunk

22 I use 'realization' in order to avoid 'actualization', which is theoretically loaded in GMR. 
of matter, $m$, does not constrain the co-realization of the possibility of making some other table from a non-overlapping piece of matter, $m *$. This intuition is silent as to whether these processes are spatiotemporally isolated or not. If it applies to any two non-overlapping processes, it applies (as a special case) to any two spatiotemporally isolated non-overlapping processes. The intuition behind these pre-theoretical constraints is, at bottom, and precisely, the Humean idea that there aren't necessary connections among different existents. So the striking nature of these necessities boils down to their being anti-Humean.

These necessary connections are exactly the same Heller identifies in Heller (2003), and which he sometimes refers to by 'inevitability relation' (and cognates):

it is inevitable that if Roz saves Righty [in one world], Lefty will die [in another world]. [...] the inevitability is still there, [and] the grounding is stronger than causation and stronger than nomological guarantee. (Heller 2003, p. 13)

Time to generalize. Both Heller (2003) and I (here so far) have worked with a simplified two-world example. In two-world totalities, which de re way one-of-the-two worlds is (according to some totality-possibility) specifically depends on which de re way theother-world is not (according to the same totality-possibility). This implication of a specific dependence is lost when we generalize. The necessary connections, however, are not lost. We still have dependence among ways of being, albeit of a generic type. ${ }^{23}$ So the situation does not improve when we generalize out of our simplified case study. ${ }^{24}$

Once we consider the real GMR-case, with infinitely many worlds (and worldpossibilities), the necessary connections would be stated with a necessity operator governing over an infinite disjunction of infinite conjunctions. That is, the necessity of the infinite disjunction of all totality-possibilities. In other terms: the worlds in the plurality must be in equilibrium so as to satisfy plenitude. As anticipated, the dependence here is generic: which qualitative way a world, w, is, generically depends on the rest of worlds dividing the labour of instantiating all the other qualitative worldways that could be instantiated. This is no less a violation of the Humean thesis, as the intrinsic character of the event of Roz saving Righty imposes an external relation of co-existence with some event of a counterpart of Roz not saving their world-mate drowning child (regardless of whether these roles are played by Lisa and Lefty or some other individuals in the plurality). According to the Humean thesis, but against the necessity of plenitude, the spatiotemporal units that constitute the totality of worlds could have run in (non-equilibrated) ways so as to leave gaps in the logical space. This is an internal tension.

Trying to escape the problem, one could insist on behalf of Lewis that the Humean thesis applies to spatiotemporally related entities only; i.e., the thesis would only speak of what arrangements of matter and distribution of properties are possible in a spatiotemporal unit. I shall make two comments against this manoeuvre.

\footnotetext{
23 See Fine (1995) and Correia (2008) on the distinction.

24 Although Heller does not generalise, the fact that the claim about there being necessary connections among different existences survives the generalisation makes his objection (in Heller 2003) immune to objections that would be based on his not generalising.
} 
First, the burden of proof is on Lewis, so the issue cannot be left (as it is) unaddressed. The claim that there are no necessary connections among spatiotemporally and causally isolated existents is at least as strongly supported by intuition as the claim there are no necessary connections among spatiotemporally and causally related existents. Heller (in the above quotation) identifies the grounds for these connections as stronger than causal and nomological. It is an urgent task for Lewis to unfold the nature of these grounds. ${ }^{25}$

Second, and most dialectically forcefully, Lewis uses the unrestricted principle of recombination against Magical Ersatzism. It is essential to Lewis' criticism against such (type of) view to make explicit that the Humean thesis is a general one:

So long as we know of no external relations except the (strictly or analogically) spatiotemporal ones, it would be good enough to stick to the special case. We can just require that spatiotemporal arrangement may vary independently of the intrinsic nature of the things arranged. [...] This is really just part of our original principle of recombination.

Once we suppose that there are further external relations, for instance the one by which the concrete world allegedly selects abstract simple elements, then, our principle of independence needs to be generalised. No matter what novel external relations there may be, it remains unintelligible that the intrinsic nature of a thing should constrain the external relations in which it stands. (181)

The generalized principle (which, as he says, is the "original principle of recombination") is, in that passage, to cover intuitions concerning the independence among a concrete entity (the actual world) and abstract ones (the Magical Ersatz worlds). In particular, it is to rule out that the (actual) intrinsic character of the world stands, necessarily, in the external relation of selection with a given element. Note, however, that what makes Lewis generalize the principle is not that the Elements are abstract, but rather that he is (safely) assuming that (as a result) they are not spatiotemporally related to the concrete world. For, if (per impossibile), despite abstract, the elements were spatiotemporally related to the concrete world, the "special case" he mentions in the first paragraph of the quote above would be contextually enough for him (that is, enough vis-à-vis his purposes of arguing against Magical Ersatizm), and no generalization would be needed. His generalisation is thus to the effect that there are no necessary connections either among spatio-temporally unrelated existences. The generalisation that generates our problem is, therefore, the same he uses against the Magical Ersatzer. As a result, there is no way to escape the internal tension without withdrawing his main objection to Magical Ersatzism, thereby compromising, again, the strength of his abductive argument for GMR. (And, as per the first comment, something should be said to explain away the (general) intuitions.)

Could the problem be nonetheless mitigated? In an attempt to do so, one could recall that, as noted above, once the case for the necessary connections is properly

\footnotetext{
25 Note also that the postulation of these necessary connections among causally isolated existents is needed only from within GMR. Any one who believed in an ontology like Lewis's, yet not in its modal relevance, would have no clear motivation to restrict recombination in the way Lewis needs it to be restricted; that is, in the (A.1)-way above.
} 
generalized, Roz saving Righty only generically depends on there being someone else who does not save another drowning child_-rather than depending specifically on, for instance, Lisa not saving Lefty. But this does not lessen the problem. Here is what immediately follows, for illustration purposes, Lewis's first statement of recombination:

Thus if there could be a dragon, and there could be a unicorn, but there couldn't be a dragon and a unicorn side by side, that would be an unacceptable gap in logical space, a failure of plenitude. And if there could be a talking head contiguous to the rest of a living human body, but there couldn't be a talking head separate from the rest of a human body, that too would be a failure of plenitude. (88)

Lewis is here illustrating the principle for the "special [intra-world] case", prior to the generalization-hence, his expressions 'side by side' and 'contiguous'. But we already know that he holds the generalized one. And his general way of talking ('a dragon', 'a unicorn', 'a talking head'...) makes it clear that even the generic form of dependence is ruled out by recombination. After all, generalized recombination sanctions, as possibilities, any arrangement of matter and distribution of properties in the totality of worlds. Yet, the necessity of plenitude blocks some of them: $a$ unicorn cannot fail to co-exist with $a$ spatiotemporally unconnected dragon. To cite Lewis against his view: that "remains unintelligible".

I contend that, to avoid this problem, Lewis should renounce the necessity of Plenitude altogether. However, and although its truth suffices to endow the account with sufficient descriptive power, this move would have high abductive costs, as briefly anticipated in Sect. 2.4.

\section{Advanced modalizing: qualifying Divers' redundancy interpretation}

As anticipated in Sect. 1, advanced modalizing is a legitimate practice within GMR but under-specified by it. So under-specified that GMR is taken to be by Hudson, if true, true but (advancedly) necessarily false and, by Divers, (advancedly) necessarily true if true. I side with Divers on the modal status of (Lewis's) GMR; and, taking the necessity of plenitude to be one of its tenets, I find that modal status problematic, for the reasons above.

My siding with Divers on the modal status of GMR does not mean I endorse his redundancy interpretation of advanced modalizing. To anticipate my reasons for dissatisfaction with it: (i) it is insensitive to the distinction between de re possibilities and (purely) qualitative ones in a way that renders it extensionally inadequate; and (ii) it objectionably neglects the force of the objections briefly surveyed in Sect. 1, unified by their puzzlement that the totality is (even if only qualitatively) necessarily the way it is.

In what follows, I will focus on Divers's (strong) reasons for his redundancy interpretation but modify the interpretation in a way that the resulting one keeps its virtues while remedying both (i) and (ii).

The following are-whether true or false—basic modal claims:

(1) It is possible that Humphrey wins the election 
(2) It is possible that Humphrey* loses the election

(3) It is necessary that Humphrey loses the election

(4) It is necessary that Humphrey* wins the election

Examples of advanced modalizing are provided by modal claims about "spatiotemporally disunified individuals, sets, numbers, properties, propositions and events" (Divers 1999, p. 217) ${ }^{26}$ :

(5) The empty set exists necessarily

(6) There could be alien properties

(7) The totality could not be (qualitatively) different

(8) It is possible that Humphrey ${ }^{\wedge}$ Humphrey* lacks a winning part $^{27}$

(9) There could be propositions that no human could think

Whether a modal claim is, from the standpoint of GMR, an instance of advanced modalizing or of basic modalizing will sometimes depend on its use. For instance, when there are quantifiers under the scope of modal vocabulary, it will depend on whether they are intended as world-restricted or not. Thus, claims (10)-(13) have both an advanced and a basic reading:

(10) It is necessary that there are talking donkeys

(11) It is necessary that there are many concrete worlds

(12) It is necessary that there is only one concrete world

(13) It is contingent that there are no talking donkeys

According to their advanced readings, they are claims about the totality of worlds (which is a spatiotemporally disunified sum). According to their basic readings, they are claims about worlds (spatiotemporally unified entities). Given the necessity of plenitude, (10) and (11) are, in GMR, true in their advanced readings but false in their basic ones, whereas it is the other way around with (12) and (13). I shall unfold the case of (12). (12) is the result of modalizing "there is only one concrete world". A GMRist can intend the quantifier in that non-modal claim as ranging over actual things only or, more widely, as ranging over everything that exists (according to her). In the former case, once this non-modal sentence is inserted as in (12), the quantifier remains as world-restricted and its content becomes: at each world, there is only one concrete world within it. And that is true. In the latter case, the quantifier remains unrestricted and its content becomes: at each world, only one concrete world exists (unrestrictedly). And that is false.

Divers (1999) makes a strong case for the redundancy interpretation of advanced modalizing in GMR which, in the case of the possibility operator, and contrasting it with the non-redundant interpretation of basic modalizing, he characterizes-in opposition to Hudson (1999)—as follows:

\footnotetext{
26 This is not neutral on the metaphysics of these sorts of entities, but this lack of neutrality is (harmlessly) allowed for the sake of illustration.

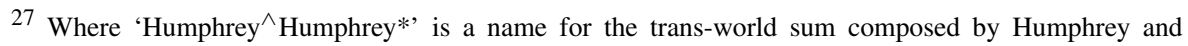
Humphrey*.
} 
Whenever the possibility operator expresses a non-trivial semantic function on quantificational sentences it is, indeed, always that of altering the scope of formerly world-restricted quantifiers. So in cases where the quantifiers were not formerly world-restricted, the possibility operator has no semantic effect on the content of the sentence within its scope. The possibility operator is semantically redundant in such a context. (Divers 1999 , p. 229)

Shortly after this characterization, he elaborates (1999, p. 230) on some truthconditional equivalences which would follow from such characterization:

Thus, restricting " $P$ " to cases expressing transworld states of affairs, we have the possibility principle:

(AP) "It is possible that $P$ " is true if and only if $P$

from which we derive each of the following:

(AN) "It is necessary that $P$ " is true if and only if $P$

(AI) "It is impossible that $P$ " is true if and only if Not $P$

(AC) "It is contingent that $P$ " is true if and only if $P$ and Not $P$

Divers' case for the redundancy interpretation is strong for several reasons, including the fact that GMR, once explicitly supplemented with such interpretation, can satisfy certain pre-theoretical desiderata, which it could not do before. ${ }^{28}$ In addition, such an interpretation keeps in line with Lewis' usage of advanced modalizing (Divers 1999, p. 231). At bottom, all these usages exploit the necessity of plenitude. As Divers notes, " $[t]$ he pattern is worth making explicit. What is true of logical space could not be otherwise, it is necessarily so and that principle is underwritten by the redundancy interpretation of necessity" (Divers 1999, p. 231).

Despite its virtues, the redundancy interpretation needs to be both expanded and qualified. The first thing to note-so far independently of the de re contingency of the totality-is that there is a gap between the redundancy interpretation as characterized in the 'whenever'-quotation above and the principles Divers arrives at shortly after. The characterization speaks of advanced modal claims whose modal vocabulary ranges over quantificational sentences, whereas the principles are said to hold for any " $P$ " that expresses a trans-world state of affairs. But some such $P$ 's are not quantificational, as (5), (7) and (8) above illustrate. The characterization of advanced modalizing, therefore, needs to be expanded so as to cover these cases too. What, then, is the semantic effect of the advanced-possibility operator on non-quantificational sentences? According to the truth-conditional equivalences Divers gets at-(AP)-(AC)—which are general enough, the modal operators would still be always semantically redundant. But that is in need of qualification, as such interpretation-given the de re contingency of the totality - renders extensionally wrong results for some P's which express a transworld states of affairs; in particular, for some $P$ 's that mention particular individuals, thereby involving de re ways for the totality to be.

\footnotetext{
28 That is, the resulting account validates the principles of modal ubiquity (MU) and possibility introduction
} (PI). 


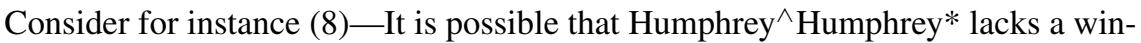
ning part. This is not only intuitively true, but also true according to GMR, provided that, as argued above, the totality is (as is theoretically desirable) de re contingent. For it is only to be expected that, according to some totality-possibility, both Humphrey and Humphrey* lose the election, and their possibility of winning is, in that totalitypossibility, represented by a third, winning counterpart of both. So according to some totality-possibility, Humphrey^ ${ }^{\wedge}$ umphrey* lacks a winning part. But because Humphrey ${ }^{\wedge}$ Humphrey* does not in fact lack a winning part, the right-to-left direction of (AN) tells us that it is not possible that Humphrey ${ }^{\wedge}$ Humphrey* lacks a winning part. So the truth of (8) constitutes, as one can immediately see via (AN), a counterexample to the principles (AP)-(AC).

Another example: Let ' $a$ ' name a non-actual counterpart of Michael Jackson who, instead of being a musician, is a shoemaker. Consider now (14):

(14) The totality of worlds could be such that Picasso is a bus driver and $a$ is a musician. $^{29}$

For analogous reasons, (14) is true in GMR but comes out as false with the general redundancy interpretation. Given that it is not the case that Picasso is a bus driver and $a$ a musician, it comes out impossible on the right-to-left direction of (AI). But according to some totality-possibility, Picasso is a bus driver and $a$ is a musician. (Among some such totality-possibilities, a counterpart of Picasso is a painter and Michael Jackson a shoemaker). So again, (14) is a counterexample to the general redundancy interpretation. ${ }^{30}$

I contend-as anticipated by (i) above-that Divers' motivation for the general redundancy interpretation has been biased by the fact that his discussion has not taken sufficiently into account de re trans-world states of affairs. And I suggest the following general truth-conditional equivalences as the simplest analysis of advanced modalizing that avoids the extensional inadequacies that afflict Divers's (general) redundancy interpretation ${ }^{31}$ :

(TP) $\nabla_{\mathrm{t}} P$ if and only if $P$ is the case according to some totality-possibility

(TN) $\square_{\mathrm{t}} P$ if and only if $P$ is the case according to all totality-possibilities

\footnotetext{
29 If preferred, one can express the same, á la (8), by means of the transworld entity Picasso $\wedge a$ 's possibility of having a part which is a bus driver and a part which is a shoemaker.

30 More examples which I shall not unfold: 'Number 2 could be my favourite number', or 'the property of being happy could have different instances'. These examples raise additional interesting issues. First, that it is not straightforward that an interpreted claim is always about either spatiotemporally unified or disunified entities. Second, it is a consequence of the de re contingency of the totality that it is contingent which set the property of being happy is. The discussion of the impact of these issues must be left for another occasion, though.

31 The introduction of advanced operators is inessential (I could have kept the un-subscripted ' $\diamond$ ' and ' $\square$ ' by prefixing the analysis, as Divers does, with something like "restricting " $P$ " to cases expressing transworld states of affairs"). I am introducing advanced operators because, as illustrated by claims (10)-(13), some $P$ 's might have both a world-restricted reading and a trans-world reading, and this will normally depend on use. By introducing advanced operators we have a syntactic resource to mark that, in the context of such operators, the (or $a$ ) transworld reading is to be selected. But these advanced operators, when translated into English, are still the ordinary 'possibility' and 'necessity'; in their advanced uses. Thanks to an anonymous referee for pressing me on this.
} 
An immediate virtue of this analysis is that, per se, it is neutral as to whether there is just one totality-possibility or (as argued in the paper) more than one, all of which qualitatively equivalent. So it is not per se incompatible with Divers' (AP)-(AC). In addition, it is easy to see that, from the standpoint of GMR, and given the necessity of plenitude, for any purely qualitative (therefore quantificational) $P$, ' $P$ ' is true in the totality if and only if ' $\square_{\mathrm{t}} P$ ' and ' $\nabla_{\mathrm{t}} P$ ' are both true. So the redundancy interpretation and the suggested alternative are co-extensional-and yet not semantically equivalent - at least for all purely qualitative $P$ 's. But if the plurality is in effect $d e$ $r e$ contingent, the current analysis behaves correctly also with cases like (8) and (14), generating correspondingly less (de re) advanced necessities and impossibilities than Divers's (AP)-(AC).

At the same time, the suggested interpretation does not neglect (from the outset) the force of objections such as that of Heller, or mine above, based on the necessary character of the totality. According to the redundancy interpretation, the claim 'there must be human beings in some world' just means that there are human beings in some world. But what is concerning, to the eyes of the objector, is not that there are human beings in some world but rather something that the redundancy interpretation doesn't even allow us to truly express: namely, the different fact of that state of affairs being necessary. (Similarly for advanced de re modal claims such as 'Humphrey must co-exist in the totality with a winning-counterpart'. What's concerning is not that he does, but that he must). On the suggested interpretation, however, advanced modal claims are quantificational statements over totality-possibilities, and what the objector finds concerning - indeed objectionable — is that more universally quantified such statements than desirable come out true in GMR. According to Heller, for instance, it leads to moral indifference that according to all totality-possibilities a counterpart of Righty drowns; according to me, that fact manifests necessary connections of a Humean kind, which reveals a tension in GMR. None of those two concerns could be grounded on the non-modal fact that a counterpart of Righty drowns, and yet this is all we can express with advanced modal claims redundantly interpreted.

Acknowledgements Earlier drafts of this paper were presented at research events in St. Andrews, Barcelona, Glasgow and Urbino. I would like to thank the audiences on all those occasions, especially including Ross Cameron, John Divers, Manuel García-Carpintero, Bob Hale, Stephan Leuenberger, Genoveva Martí, Manuel Pérez-Otero, and Crispin Wright. Special thanks are due to Claudio Calosi, my commentator at the Urbino conference (Another World is Possible) and whose penetrating remarks helped me to reconstruct my points in a clearer way. I am also greatly thankful to Marianna Antonutti and Pierluigi Graziani-the volume editors—and a number of anonymous referees for this journal.

Open Access This article is distributed under the terms of the Creative Commons Attribution 4.0 International License (http://creativecommons.org/licenses/by/4.0/), which permits unrestricted use, distribution, and reproduction in any medium, provided you give appropriate credit to the original author(s) and the source, provide a link to the Creative Commons license, and indicate if changes were made.

\section{References}

Adams, R. (1974). Theories of actuality. Noûs, 8(3), 211-231.

Beedle, A. (1996). Modal fatalism. The Philosophical Quarterly, 46(185), 488-495. 
Correia, F. (2008). Ontological dependence. Philosophy Compass, 3(5), 1013-1032.

Divers, J. (1999). A genuine realist theory of advanced modalizing. Mind, 108(430), 217-240.

Fine, K. (1995). Ontological dependence. Proceedings of the Aristotelian Society, 95, 269-290.

Heller, M. (2003). The immorality of Modal Realism, or: How I learned to stop worrying and let the children drown. Philosophical Studies, 114, 1-22.

Hudson, H. (1997). Brute facts. Australasian Journal of Philosophy, 75(1), 77-82.

Hudson, H. (1999). A true necessary falsehood. Australasian Journal of Philosophy, 77(1), 89-91.

Lewis, D. (1986). On the plurality of worlds. Oxford: Blackwell.

Ridenour, R. (2000). An essentialist theory of modality. Ann Arbor: UMI Microform.

Skyrms, B. (1976). Possible worlds, physics and metaphysics. Philosophical Studies, 30, 323-332.

Yagisawa, T. (1988). Beyond possible worlds. Philosophical Studies, 54, 175-204. 